\title{
Upper Bounds to the Ground State Energies of the One- and Two-Component Charged Bose Gases
}

\author{
Jan Philip Solovej* \\ Institute for Mathematical Sciences ${ }^{\dagger}$ \\ University of Copenhagen \\ Universitetsparken 5 \\ DK-2100 Copenhagen \\ DENMARK. \\ e-mail: solovej@math.ku.dk
}

\begin{abstract}
We prove upper bounds on the ground state energies of the one- and two-component charged Bose gases. The upper bound for the one-component gas agrees with the high density asymptotic formula proposed by L. Foldy in 1961. The upper bound for the twocomponent gas agrees in the large particle number limit with the asymptotic formula conjectured by F. Dyson in 1967. Matching asymptotic lower bounds for these systems were proved in references [10] and [11. The formulas of Foldy and Dyson which are based on Bogolubov's pairing theory have thus been validated.
\end{abstract}

\section{Introduction and main results}

In 1961 L. Foldy [7] used Bogolubov's 1947 pairing theory [4] for Bose systems to give a heuristic calculation of the ground state energy of a one-component charged Bose gas in the

*Work partially supported by NSF grant DMS-0111298, by EU grant HPRN-CT-2002-00277, by MaPhySto - A Network in Mathematical Physics and Stochastics, funded by The Danish National Research Foundation, and by grants from the Danish research council.

$\dagger$ Most of this work was done while the author was visiting the School of Mathematics, Institute for Advanced Study, Princeton.

(C)2004 by the author. This article may be reproduced in its entirety for non-commercial purposes. 
high density limit. The one-component Bose gas is a system of Bose particles all of the same charge moving in the presence of a fixed uniform background of the opposite charge.

In 1967 F. Dyson [6] considered the two-component Bose gas with two species of bosons with opposite charges. Motivated by Foldy's calculation Dyson was able to prove a rigorous upper bound on the ground state energy. A famous consequence of Dyson's upper bound is that charged bosonic matter is not stable, the ground state energy is super-linear in the number of particles. Dyson, moreover, conjectured an exact asymptotic form of the ground state energy in the limit of a large number of particles.

In [10] it was proved that Foldy's calculation is indeed correct as a leading asymptotic lower bound for the ground state energy of the one-component charged Bose gas in the high density limit.

In [11] it was similarly proved that Dyson's conjectured expression is correct as an asymptotic lower bound for the ground state energy of the two-component charged Bose gas in the limit of a large number of particles.

The aim of the present paper is to prove the corresponding upper bounds thus validating both Foldy's one-component and Dyson's two-component formulas.

It should be mentioned that Foldy's calculation may be viewed as a trial state calculation and may thus be turned into a rigorous upper bound. Foldy, however, uses periodic boundary conditions, and a periodic version of the Coulomb potential. It is not known whether this formulation has the same thermodynamic limit as the formulation given below.

The one-component Bose gas is a system of $N$ particles all of the same charge +1 , say, constrained to a box $\Lambda=[0, L]^{3} \subset \mathbb{R}^{3}$, in which there is a uniform background charge of density $\rho$.

The Hamiltonian for the one-component charged Bose gas is thus

$$
H_{N}^{(1)}=\sum_{i=1}^{N}\left(-\frac{1}{2} \Delta_{i}-V\left(x_{i}\right)\right)+\sum_{1 \leq i<j \leq N}\left|x_{i}-x_{j}\right|^{-1}+C
$$

where

$$
V(x)=\rho \int_{\Lambda}\left|x_{i}-y\right|^{-1} \mathrm{~d} y, \quad C=\frac{\rho^{2}}{2} \iint_{\Lambda \times \Lambda}|x-y|^{-1} \mathrm{~d} x \mathrm{~d} y .
$$

We use Dirichlet boundary conditions.

It is known from the work of Lieb and Narnhofer [9] that the ground state energy $E^{(1)}(N)$ of $H_{N}^{(1)}$ has a thermodynamic limit if we restrict to a neutral system

$$
e(\rho)=\lim _{\substack{N \rightarrow \infty \\ L^{3}=N / \rho}} \frac{E^{(1)}(N)}{L^{3}} .
$$


It is however also shown in 9] that one will get the same thermodynamic energy by minimizing over all particle numbers, i.e.,

$$
e(\rho)=\lim _{L \rightarrow \infty} \inf _{N} \frac{E^{(1)}(N)}{L^{3}} .
$$

THEOREM 1.1 (Foldy's formula). The ground state energy $e(\rho)$ of the one-component charged Bose gas satisfies the asymptotics

$$
\lim _{\rho \rightarrow \infty} \rho^{-5 / 4} e(\rho)=-I_{0}
$$

where

$$
I_{0}=(2 / \pi)^{3 / 4} \int_{0}^{\infty} 1+x^{4}-x^{2}\left(x^{4}+2\right)^{1 / 2} \mathrm{~d} x=\frac{4^{5 / 4} \Gamma(3 / 4)}{5 \pi^{1 / 4} \Gamma(5 / 4)} .
$$

The two component Bose gas is described by the Hamiltonian

$$
H_{N}^{(2)}=\sum_{i=1}^{N}-\frac{1}{2} \Delta_{i}+\sum_{1 \leq i<j \leq N} \frac{e_{i} e_{j}}{\left|x_{i}-x_{j}\right|}
$$

acting on the Hilbert space $L^{2}\left(\mathbb{R}^{3} \times\{1,-1\}\right)$, where the variable $\left(x_{i}, e_{i}\right) \in \mathbb{R}^{3} \times\{1,-1\}$ gives the position and charge of particle $i$.

The word two component refers to the fact that the charge of each particle can be either positive or negative. Thus the gas has a positive and a negative component. One would not normally consider the charges as variables, but rather fix them to have given values. If we did that, the Hamiltonian would not be fully symmetric in all $N$ variables, but only in the variables for the positively charged particles and negatively charged particles separately. Clearly, the charge variables commute with the Hamiltonian and the bottom of the spectrum (the ground state energy) $E^{(2)}(N)$ of $H_{N}^{(2)}$ will therefore be achieved for a fixed combination of charges (rather than a superposition).

THEOREM 1.2 (Dyson's formula). The ground state energy $E^{(2)}(N)$ of the two-component charged Bose gas satisfies the asymptotics

$$
\lim _{N \rightarrow \infty} N^{-7 / 5} E^{(2)}(N)=-A
$$

where $A$ is the positive constant determined by the variational principle

$$
-A=\inf \left\{\frac{1}{2} \int_{\mathbb{R}^{3}}|\nabla \Phi|^{2}-I_{0} \int_{\mathbb{R}^{3}} \Phi^{5 / 2} \mid 0 \leq \Phi, \int_{\mathbb{R}^{3}} \Phi^{2}=1\right\}
$$

with $I_{0}$ again given by (3). 
In [6] Dyson proves that $E^{(2)}(N) \leq-C N^{7 / 5}$, but with a constant different from $A$. He conjectures that the correct value is given as above. That the exponent $7 / 5$ is, indeed, correct was first proved in 1988 by Conlon, Lieb, and Yau in [5], where they show a lower bound $-C N^{7 / 5}$, but still not with the correct constant. They also proved that $5 / 4$ is the correct exponent in Foldy's formula. The asymptotic lower bounds in Theorems 1.1 and 1.2 were proved in [10] and [1] respectively. The main results of the following paper are the asymptotic upper bounds.

In Sect. 2 we give a general construction of bosonic trial states on the bosonic Fock space over a general Hilbert space. The trial states will be build from coherent states and squeezed states. The trial states are essentially the ones dictated by Bogolubov theory. These trial states are the bosonic equivalent of the fermionic states in Hartree-Fock theory or rather to their extension including the Bardeen-Cooper-Schrieffer states (see [1]).

In the same way as fermionic systems may be approximated by the semi-classical ThomasFermi theory we will also use a semi-classical type approximation to the Bogolubov trial states.

In Sect. 3 we use the general trial state method to give an upper bound on the ground state energy for the two-component gas, but in a grand canonical setting where we do not fix the total number of particles.

In Sect 3.1 we show how to get an upper bound for fixed particle number and thus prove Theorem 1.2 .

In Sect. 4 we use the general trial state method to give an upper bound on the ground state energy for the one-component gas and prove Theorem 1.1.

A key ingredient in the proofs is a semiclassical construction where we represent operators as phase-space integrals with coherent states symbols and use the Berezin-Lieb inequalities. We need an operator version of the inequality. This is discussed in Appendix A.

Acknowledgment: I would like to thank Elliott Lieb, Kumar Raman, and Robert Seiringer for valuable discussions.

\section{The abstract trial state construction}

Our goal in this section is to construct trial states on the bosonic Fock space $\mathcal{F}=\mathcal{F}\left(\mathcal{H}_{1}\right)=$ $\bigoplus_{N=0}^{\infty} \mathcal{H}_{N}$, over some Hilbert Space $\mathcal{H}_{1}$, i.e., $\mathcal{H}_{N}=\bigotimes_{\text {Sym }}^{N} \mathcal{H}_{1}$ and $\mathcal{H}_{0}=\mathbb{C}$. We will be using the language of bosonic creation and annihilation operators as a convenient tool for the book keeping. We denote by $|0\rangle$ the vacuum vector in $\mathcal{F}$. If $T$ is an operator on $\mathcal{H}_{1}$ and $W$ is an operator $\mathcal{H}_{1} \otimes \mathcal{H}_{1}$, which is symmetric under interchange of the tensor factors, we may lift 
(also referred to as second quantize) these operators to $\mathcal{F}$ as

$$
\bigoplus_{N=1}^{\infty} \sum_{i=1}^{N} T_{i} \text { and } \bigoplus_{N=2}^{\infty} \sum_{1 \leq i<j \leq N} W_{i j}
$$

Here $T_{i}$ refers to the operator $T$ acting on the $i$ th factor in the tensor product and $W_{i j}$ refers to $W$ acting on the $i$ th and $j$ th factors. If $u_{\alpha}, \alpha=1, \ldots$ is an orthonormal basis for $\mathcal{H}_{1}$ we can express these operators using creation and annihilation operators as

$$
\bigoplus_{N=1}^{\infty} \sum_{i=1}^{N} T_{i}=\sum_{\alpha, \beta}\left(u_{\alpha}, T u_{\beta}\right) a\left(u_{\alpha}\right)^{*} a\left(u_{\beta}\right)
$$

and

$$
\bigoplus_{N=0}^{\infty} \sum_{1 \leq i<j \leq N} W_{i j}=\frac{1}{2} \sum_{\alpha \beta \mu \nu}\left(u_{\alpha} \otimes u_{\beta}, W u_{\mu} \otimes u_{\nu}\right) a\left(u_{\alpha}\right)^{*} a\left(u_{\beta}\right)^{*} a\left(u_{\nu}\right) a\left(u_{\mu}\right) .
$$

Of special interest is the number operator (the second quantization of the identity)

$$
\mathcal{N}=\bigoplus_{N=0}^{\infty} N
$$

If $\phi \in \mathcal{H}_{1}$ is a not necessarily normalized vector we define the corresponding coherent state as the normalized vector in Fock space

$$
\begin{aligned}
|\phi\rangle_{\mathrm{C}} & =\exp \left(-\|\phi\|^{2} / 2+a(\phi)^{*}\right)|0\rangle \\
& =\sum_{n=0}^{\infty} e^{-\|\phi\|^{2} / 2} \frac{\left(a(\phi)^{*}\right)^{n}}{n !}|0\rangle,
\end{aligned}
$$

and for a normalized $\psi \in \mathcal{H}_{1}$ we define the squeezed state depending on $\lambda \in \mathbb{C}$ with $|\lambda|<1$

$$
\begin{aligned}
|\lambda ; \psi\rangle_{\mathrm{S}} & =\left(1-|\lambda|^{2}\right)^{1 / 4} \exp \left(-(\lambda / 2) a(\psi)^{*} a(\psi)^{*}\right)|0\rangle \\
& =\left(1-|\lambda|^{2}\right)^{1 / 4} \sum_{n=0}^{\infty} \frac{(-\lambda / 2)^{n}}{n !}\left(a(\psi)^{*}\right)^{2 n}|0\rangle .
\end{aligned}
$$

It is straightforward to check that these states are normalized. Up to an overall phase $|\phi\rangle_{\mathrm{C}}$ and $|\lambda ; \psi\rangle_{\mathrm{S}}$ are characterized by

$$
\left(a(\phi)-\|\phi\|^{2}\right)|\phi\rangle_{\mathrm{C}}=0 \quad \text { and } \quad\left(a(\psi)+\lambda a(\psi)^{*}\right)|\lambda ; \psi\rangle_{\mathrm{S}}=0
$$

We immediately see that

$$
{ }_{\mathrm{C}}\left\langle\phi\left|\left(a(\phi)^{*}\right)^{m} a(\phi)^{k}\right| \phi\right\rangle_{\mathrm{C}}=\|\phi\|^{2(m+k)} .
$$


For the squeezed state we get

$$
\begin{aligned}
{ }_{\mathrm{S}}\langle\lambda ; \psi| & \left(a(\psi)^{*}\right)^{j} a(\psi)^{j+2 k}|\lambda ; \psi\rangle_{\mathrm{S}} \\
= & \left(1-|\lambda|^{2}\right)^{1 / 2} \sum_{n=0}^{\infty} \frac{(2 n+2 k) !}{(n+k) !^{2}}(2 n-j+1)(2 n-j+2) \cdots(2 n) \\
& \times(n+k)(n+k-1) \cdots(n+1)(|\lambda| / 2)^{2 n}(-\lambda / 2)^{k} \\
= & \left(1-|\lambda|^{2}\right)^{1 / 2}|\lambda|^{j}(-\lambda)^{k} \frac{d^{j}}{d|\lambda|^{j}}\left(|\lambda|^{-1} \frac{d}{d|\lambda|}\right)^{k}\left(1-|\lambda|^{2}\right)^{-1 / 2} .
\end{aligned}
$$

Moreover, the expectation in the state $|\lambda ; \psi\rangle_{\mathrm{S}}$ of a product of an odd number of the operators $a(\psi)^{*}$ or $a(\psi)$ vanishes.

For the expectation of the particle number we find

$$
{ }_{\mathrm{C}}\left\langle\phi\left|a(\phi /\|\phi\|)^{*} a(\phi /\|\phi\|)\right| \phi\right\rangle_{\mathrm{C}}=\|\phi\|^{2} \quad \text { and } \quad{ }_{\mathrm{S}}\left\langle\lambda ; \psi\left|a(\psi)^{*} a(\psi)\right| \lambda ; \psi\right\rangle_{\mathrm{S}}=\frac{|\lambda|^{2}}{1-|\lambda|^{2}} .
$$

We point out that the variation in the particle number is very different in the coherent state and in the squeezed state

$$
\begin{aligned}
{ }_{\mathrm{C}}\left\langle\phi\left|\left(a(\phi /\|\phi\|)^{*} a(\phi /\|\phi\|)\right)^{2}\right| \phi\right\rangle_{\mathrm{C}}-{ }_{\mathrm{C}}\left\langle\phi\left|a(\phi /\|\phi\|)^{*} a(\phi /\|\phi\|)\right| \phi\right\rangle_{\mathrm{C}}^{2} & =\|\phi\|^{2} \\
{ }_{\mathrm{S}}\left\langle\lambda ; \psi\left|\left(a(\psi)^{*} a(\psi)\right)^{2}\right| \lambda ; \psi\right\rangle_{\mathrm{S}}-{ }_{\mathrm{S}}\left\langle\lambda ; \psi\left|a(\psi)^{*} a(\psi)\right| \lambda ; \psi\right\rangle_{\mathrm{S}}^{2} & =\frac{2|\lambda|^{2}}{\left(1-|\lambda|^{2}\right)^{2}} .
\end{aligned}
$$

Thus in the coherent state the standard deviation of the particle number is the square root of the expectation itself, whereas for the squeezed state the standard deviation of the particle number is, in fact, greater than the expectation itself. For this reason the squeezed states are not appropriate for describing Bose condensates with a macroscopic and sharply defined occupation number in a specific one-particle state. To describe condensates we will use coherent states.

We will here define a variational principle corresponding to the Bogolubov theory of Bose gases. We shall do this by characterizing the set of variational trial states.

The Bogolubov variational theory is very similar to the Hartree-Fock theory for Fermi gases. More precisely, it is similar to the generalized Hartree-Fock theory which includes the Bardeen-Cooper-Schrieffer (BCS) trial states. In generalized Hartree-Fock theory (see [1]) the class of trial states is defined to be the quasi-free states on a fermionic Fock space. For the ground state (zero temperature) theory we may restrict to pure quasi-free states.

To describe the variational states of Bogolubov theory we we will again start from (normalized) quasi-free pure states. Such a state may be characterized as follows. If $\Psi \in \mathcal{F}\left(\mathcal{H}_{1}\right)$ is a normalized quasi-free pure states there exists an orthonormal family $\psi_{1}, \ldots$ of $\mathcal{H}_{1}$ and a sequence of numbers $0<\lambda_{1}, \ldots<1$ with $\sum_{\alpha=1}^{\infty} \lambda_{\alpha}^{2}<\infty$ such that

$$
\Psi=\prod_{\alpha=1}\left[\left(1-\lambda_{\alpha}^{2}\right)^{\frac{1}{4}} \exp \left(-\frac{\lambda_{\alpha}}{2} a\left(\psi_{\alpha}\right)^{*} a\left(\psi_{\alpha}\right)^{*}\right)\right]|0\rangle
$$


A straightforward but lengthy calculation from (11) shows that the quasi-free state satisfies

$$
\begin{aligned}
\left(\Psi, a_{1}^{\sharp} a_{2}^{\sharp} a_{3}^{\sharp} a_{4}^{\sharp} \Psi\right)= & \left(\Psi, a_{1}^{\sharp} a_{2}^{\sharp} \Psi\right)\left(\Psi, a_{3}^{\sharp} a_{4}^{\sharp} \Psi\right)+\left(\Psi, a_{1}^{\sharp} a_{4}^{\sharp} \Psi\right)\left(\Psi, a_{2}^{\sharp} a_{3}^{\sharp} \Psi\right) \\
& +\left(\Psi, a_{1}^{\sharp} a_{3}^{\sharp} \Psi\right)\left(\Psi, a_{2}^{\sharp} a_{4}^{\sharp} \Psi\right)
\end{aligned}
$$

and from the definition of the state we have for all integers $m \geq 1$

$$
\left(\Psi, a_{1}^{\sharp} \cdots a_{2 m-1}^{\sharp} \Psi\right)=0 .
$$

In (15) and (16) $, a_{j}^{\sharp}, j=1,2 \ldots$ refer to any creation or annihilation operators. The relation (15) is the case $m=2$ of the more general rule

$$
\left(\Psi, a_{1}^{\sharp} \cdots a_{2 m}^{\sharp} \Psi\right)=\sum_{\pi \in P_{2 m}}\left(\Psi, a_{\pi(1)}^{\sharp} a_{\pi(2)}^{\sharp} \Psi\right) \cdots\left(\Psi, a_{\pi(2 m-1)}^{\sharp} a_{\pi(2 m)}^{\sharp} \Psi\right),
$$

where $P_{2 m}$ is the set of pairing permutations

$$
\begin{array}{r}
P_{2 m}=\left\{\pi \in S_{2 m} \mid \pi(2 j-1)<\pi(2 j+1), j=1, \ldots, m-1\right. \\
\pi(2 j-1)<\pi(2 j), j=1, \ldots, m\} .
\end{array}
$$

We shall here use this only in the case (15) when $m=2$.

The one-particle density matrix of the quasi-free state $\Psi$ is the operator $\gamma_{1}$ defined on the one-body space $\mathcal{H}_{1}$ by $\left(g, \gamma_{1} f\right)_{\mathcal{H}_{1}}=\left(\Psi, a(f)^{*} a(g) \Psi\right)_{\mathcal{F}}$ where $f, g \in \mathcal{H}_{1}$. From (111)

$$
\gamma_{1}=\sum_{\alpha=1}^{\infty} \frac{\lambda_{\alpha}^{2}}{1-\lambda_{\alpha}^{2}}\left|\psi_{\alpha}\right\rangle\left\langle\psi_{\alpha}\right|
$$

Note, in particular, that the one-particle density matrix is a positive semi-definite trace class operator with

$$
\operatorname{Tr} \gamma_{1}=(\Psi, \mathcal{N} \Psi)=\sum_{\alpha=1}^{\infty} \frac{\lambda_{\alpha}^{2}}{1-\lambda_{\alpha}^{2}}<\infty
$$

Connected to the quasi-free pure state $\Psi$ we also have the symmetric bilinear form $\xi_{1}$ on $\mathcal{H}_{1}$ given by $\xi_{1}(f, g)=\left(\Psi, a(f)^{*} a(g)^{*} \Psi\right)_{\mathcal{F}}$. We find, again from (11), that

$$
\xi_{1}(f, g)=\sum_{\alpha=1}^{\infty} \frac{-\lambda_{\alpha}}{1-\lambda_{\alpha}^{2}}\left(\psi_{\alpha}, f\right)\left(\psi_{\alpha}, g\right)
$$

We may identify $\xi_{1}$ with a linear map $\xi_{1}: \mathcal{H}_{1} \rightarrow \mathcal{H}_{1}^{*}$, from the one-body space $\mathcal{H}_{1}$ to its dual space $\mathcal{H}_{1}^{*}$. We then have the relations

$$
\xi_{1}^{*} \xi_{1}=\gamma_{1}\left(\gamma_{1}+1\right) \quad \xi_{1} \gamma_{1}=\gamma_{1} \xi_{1}
$$


where we have also identified $\gamma_{1}$ in the natural way with a map from $\mathcal{H}_{1}^{*}$ to itself. If we introduce the operator $\Gamma: \mathcal{H}_{1} \oplus \mathcal{H}_{1}^{*} \rightarrow \mathcal{H}_{1} \oplus \mathcal{H}_{1}^{*}$ defined using matrix notation as

$$
\Gamma=\left(\begin{array}{cc}
\gamma_{1} & \xi_{1} \\
\xi_{1}^{*} & 1+\gamma_{1}
\end{array}\right)
$$

we may rewrite the condition (211) as

$$
\Gamma\left(\begin{array}{cc}
-1 & 0 \\
0 & 1
\end{array}\right) \Gamma=\Gamma .
$$

We may refer to an operator satisfying this condition as a symplectic projection. In the fermionic case the corresponding operator is simply a projection. Note that the operator $\Gamma$ may also be described by

$$
\left(\left|f_{1}\right\rangle \oplus\left\langle g_{1}|, \Gamma| f_{2}\right\rangle \oplus\left\langle g_{2}\right|\right)_{\mathcal{H}_{1} \oplus \mathcal{H}_{1}^{*}}=\left(\Psi,\left(a\left(f_{2}\right)^{*}+a\left(g_{2}\right)\right)\left(a\left(f_{1}\right)+a\left(g_{1}\right)^{*}\right) \Psi\right)_{\mathcal{F}\left(\mathcal{H}_{1}\right)}
$$

where we have used the Dirac bra and ket notation to denote elements of $\mathcal{H}_{1}$ and $\mathcal{H}_{1}^{*}$ respectively.

Given a positive definite trace class operator $\gamma_{1}$ and a symmetric bilinear form $\xi_{1}$ satisfying (21) we may find a unique quasi-free pure state $\Psi$ such that $\gamma_{1}$ is the corresponding oneparticle density matrix and $\xi_{1}$ the corresponding bilinear form. To see this one simply has to show that there exists an orthonormal family $\psi_{1}, \ldots$ and a sequence of positive numbers $\lambda_{1}, \ldots$ such that (19) and (20) hold. This is a fairly simple exercise in linear algebra.

The choice of $\xi_{1}$ is equivalent to a particular choice of eigenbasis for $\gamma_{1}$. If $\gamma_{1}$ has real eigenfunctions (in some representation) there is a particular $\xi_{1}$ corresponding to this choice of basis. We shall use this in our construction of states in the next sections.

Consider as an example $\gamma_{1}$ being a real translation invariant operator on the Hilbert space $L^{2}\left(\mathbb{R}^{n} / 2 \pi \mathbb{Z}^{n}\right)$ of square integrable functions on the torus. The real eigenfunctions come in degenerate pairs of the form $\cos (p x)$ and $\sin (p x), p \in \mathbb{Z}^{n}$. The associated quasi-free state will in the exponent have terms of the form

$$
a(\cos (p x))^{*} a(\cos (p x))^{*}+a(\sin (p x))^{*} a(\sin (p x))^{*}=a\left(e^{i p x}\right)^{*} a\left(e^{-i p x}\right)^{*}
$$

This corresponds to a pairing of states with opposite momenta, as is the usual case in the Bogolubov pair theory.

The Bogolubov variational states are not just quasi-free states as defined above. In fact, quasi-free states being build out of squeezed states are not well suited for describing condensates (see the discussion after (12) and (13). We introduce condensates by appropriate unitary transformations of quasi-free states as we shall now describe. 
Given $\phi \in \mathcal{H}_{1}$ we have a unitary map $U_{\phi}$ on the Fock space $\mathcal{F}\left(\mathcal{H}_{1}\right)$ which satisfies

$$
U_{\phi}^{*} a(f) U_{\phi}=a(f)+(f, \phi) .
$$

This unitary is unique up to an overall complex phase, which we may fix by noting that we can add the requirement that the unitary maps the vacuum state to a a coherent state

$$
U_{\phi}|0\rangle=|\phi\rangle_{\mathrm{C}} .
$$

From the first identity in (9) it is clear that $U_{\phi}$ satisfies this up to a phase.

The Bogolubov variational states are constructed from a quasi-free state $\Psi$ and a vector $\phi \in \mathcal{H}_{1}$ as $\Psi_{\phi}=U_{\phi} \Psi$. From the above discussion we see that a Bogolubov state may be described as follows.

DEFINITION 2.1 (Bogolubov variational states). A Bogolubov state on the bosonic Fock space $\mathcal{F}\left(\mathcal{H}_{1}\right)$ is given by

$$
\Psi_{\phi, \gamma_{1}, \xi_{1}}=\prod_{\alpha=1}\left[\left(1-\lambda_{\alpha}^{2}\right)^{\frac{1}{4}} \exp \left(-\frac{\lambda_{\alpha}}{2}\left(a\left(\psi_{\alpha}\right)^{*}-\left(\phi, \psi_{\alpha}\right)\right)\left(a\left(\psi_{\alpha}\right)^{*}-\left(\phi, \psi_{\alpha}\right)\right)\right)\right]|\phi\rangle_{\mathrm{C}} .
$$

where $\phi \in \mathcal{H}_{1}$ and $\psi_{1}, \psi_{2} \ldots$ is an orthonormal family in $\mathcal{H}_{1}$ and $0<\lambda_{1}, \lambda_{2}, \ldots<1$ satisfy $\sum_{\alpha=1}^{\infty} \lambda_{\alpha}^{2}=1$. We call $\phi$ the condensate vector and $\psi_{1}, \psi_{2} \ldots$ the pair states.

There is a one-to-one correspondence between Bogolubov states and triples $\left(\phi, \gamma_{1}, \xi_{1}\right)$ consisting of a vector $\phi \in \mathcal{H}_{1}$ a positive trace class operator $\gamma_{1}$ on $\mathcal{H}_{1}$ and a bilinear form $\xi_{1}$ on $\mathcal{H}_{1} \times \mathcal{H}_{1}$ satisfying (21). The correspondence is given by (191) and (201).

We find for the one-particle density matrix of the Bogolubov state $\Psi_{\phi, \gamma_{1}, \xi_{1}}$ that

$$
\begin{aligned}
\left(\Psi_{\phi, \gamma_{1}, \xi_{1}}, a(u)^{*} a(v) \Psi_{\phi, \gamma_{1}, \xi_{1}}\right)_{\mathcal{F}\left(\mathcal{H}_{1}\right)} & =\left(\Psi_{0, \gamma_{1}, \xi_{1}},\left(a(u)^{*}+(\phi, u)\right)(a(v)+(v, \phi)) \Psi_{0, \gamma_{1}, \xi_{1}}\right)_{\mathcal{F}\left(\mathcal{H}_{1}\right)} \\
& =\left(v, \gamma_{1} u\right)+(v, \phi)(\phi, u)
\end{aligned}
$$

and likewise for the two-particle density matrix using (15)

$$
\begin{aligned}
& \left(\Psi_{\phi, \gamma_{1}, \xi_{1}}, a\left(u_{1}\right)^{*} a\left(u_{2}\right)^{*} a\left(v_{2}\right) a\left(v_{1}\right) \Psi_{\phi, \gamma_{1}, \xi_{1}}\right)_{\mathcal{F}\left(\mathcal{H}_{1}\right)}=\left(v_{1}, \phi\right)\left(v_{2}, \phi\right)\left(\phi, u_{1}\right)\left(\phi, u_{2}\right) \\
& \quad+\xi_{1}\left(u_{1}, u_{2}\right)\left(v_{1}, \phi\right)\left(v_{2}, \phi\right)+\overline{\xi_{1}\left(v_{1}, v_{2}\right)}\left(\phi, u_{1}\right)\left(\phi, u_{2}\right) \\
& \quad+\left(v_{2}, \gamma_{1} u_{1}\right)\left(v_{1}, \phi\right)\left(\phi, u_{2}\right)+\left(v_{1}, \gamma_{1} u_{2}\right)\left(v_{2}, \phi\right)\left(\phi, u_{1}\right) \\
& \quad+\left(v_{2}, \gamma_{1} u_{2}\right)\left(v_{1}, \phi\right)\left(\phi, u_{1}\right)+\left(v_{1}, \gamma_{1} u_{1}\right)\left(v_{2}, \phi\right)\left(\phi, u_{2}\right) \\
& \quad+\left(v_{1}, \gamma_{1} u_{1}\right)\left(v_{2}, \gamma_{1} u_{2}\right)+\left(v_{1}, \gamma_{1} u_{2}\right)\left(v_{2}, \gamma_{1} u_{1}\right)+\overline{\xi_{1}\left(v_{1}, v_{2}\right)} \xi_{1}\left(u_{1}, u_{2}\right) .
\end{aligned}
$$

The above trial states are motivated by the Bogolubov approximation for Bose condensed systems. The states $\phi$ represents the condensate, whereas the states $\psi_{\alpha}, \alpha=1, \ldots$ represent 
the pair states. A key ingredient in the Bogolubov approximation is the c-number substitution, i.e., the replacement of the operator $a(\phi)$ by the number $\|\phi\|^{2}$. This replacement will give the correct value for expectations of normal ordered products in the Bogolubov states if we have the additional assumption that $\gamma_{1} \phi=0$ (see (10). In Section 3 we will choose a Bogolubov state satisfying this assumption, but in Section 4 the Bogolubov state that we choose will not satisfy the assumption.

It is not the aim here to study the general properties of the Bogolubov variational problem, i.e., the minimization of the expectation of many-body Hamiltonians restricted to Bogolubov states. We will instead proceed to the specific examples of the one-component and two-component charged Bose gas. Here we shall not characterize the exact Bogolubov minimizer, but instead give the semiclassical approximations to these states which give the leading order asymptotics in Theorems 1.1 and 1.2 .

The Hamiltonians that we are interested in are particle number conserving, i.e., commute with particle number and the reader may wonder why we do not define a class of particle conserving, i.e., canonical trial states rather than the grand canonical states above. As in the fermionic BCS theory it is very complicated to write a canonical trial state. The calculations are greatly simplified in the grand canonical setting. Simple minded trial states with a fixed number of particles in the condensate will not give the correct approximation, since the important virtual pair creation will be lost.

\section{The two-component charged Bose gas}

We consider the two component Bose gas described by the Hamiltonian

$$
H^{(2)}=\bigoplus_{N=0}^{\infty} H_{N}^{(2)}, \quad H_{N}^{(2)}=\sum_{i=1}^{N}-\frac{1}{2} \Delta_{i}+\sum_{1 \leq i<j \leq N} \frac{e_{i} e_{j}}{\left|x_{i}-x_{j}\right|}
$$

acting on the Fock space $\mathcal{F}\left(L^{2}\left(\mathbb{R}^{3} \times\{1,-1\}\right)\right.$, where the variable $\left(x_{i}, e_{i}\right) \in \mathbb{R}^{3} \times\{1,-1\}$ gives the position and charge of particle $i$.

Our goal here is first to construct a grand canonical normalized trial function

$$
\Psi \in \mathcal{F}\left(L^{2}\left(\mathbb{R}^{3} \times\{1,-1\}\right)\right.
$$

with particle numbers concentrated sharply around the average value $\langle\mathcal{N}\rangle=(\Psi, \mathcal{N} \Psi)$ and such that

$$
\left\langle H^{(2)}\right\rangle=\left(\Psi, H^{(2)} \Psi\right) \leq-A\langle\mathcal{N}\rangle^{7 / 5}+o\left(\langle\mathcal{N}\rangle^{7 / 5}\right)
$$


for large $\langle\mathcal{N}\rangle$. We have denoted the expectation in the state $\Psi$ by $\langle\mathcal{A}\rangle=(\Psi, \mathcal{A} \Psi)$. From this the proof of Dyson's formula Theorem 1.2 (i.e., the fact that we can achieve this estimate with a trial function of fixed particle number) will follow fairly easily (see Section 3.11).

To construct the trial state $\Psi$ we use the method from the previous section. We begin with a normalized minimizer $\Phi$ for the variational problem (4). Using spherically symmetric decreasing rearrangements it is not difficult to see that a minimizer exists and that it may be chosen positive and spherically symmetric decreasing. Moreover, from the Euler-Lagrange equation it is exponentially decreasing and smooth. It is, however, not essential that we can find an exact minimizer with these properties. As we shall see, we could as well have chosen an approximate minimizer, which is smooth and compactly supported.

Let $n>0$ and define the normalized function

$$
\phi_{0}(x)=n^{3 / 10} \Phi\left(n^{1 / 5} x\right) .
$$

We define a normalized state $\Psi_{n} \in \mathcal{F}$ as in (22) with the condensate vector on $L^{2}\left(\mathbb{R}^{3} \times\right.$ $\{-1,1\})$ given by

$$
\phi(x, e)=\sqrt{\frac{n}{2}} \phi_{0}(x)
$$

and the operator $\gamma_{1}$ on $L^{2}\left(\mathbb{R}^{3} \times\{-1,1\}\right)$ defined by the integral kernel

$$
\gamma_{1}\left(x, e ;, y, e^{\prime}\right)=\frac{1}{2} \gamma(x, y) e e^{\prime}
$$

where $\gamma$ is a positive semi-definite trace class operator having real eigenfunctions. We shall make an explicit choice for $\gamma$ below (see 39). We write the spectral decomposition of $\gamma$ as

$$
\gamma=\sum_{\alpha=1}^{\infty} \frac{\lambda_{\alpha}^{2}}{1-\lambda_{\alpha}^{2}}\left|\psi_{\alpha}\right\rangle\left\langle\psi_{\alpha}\right|
$$

where $\psi_{\alpha}, \alpha=1, \ldots$ is a real orthonormal basis and $0 \leq \lambda_{\alpha}<1$ for $\alpha=1, \ldots$ Observe that on the space $L^{2}\left(\mathbb{R}^{3} \times\{1,-1\}\right)$ we have $\|\phi\|^{2}=n$ and $\gamma_{1} \phi=0$. Denoting

$$
\psi_{\alpha \pm}(x, e)=\psi_{\alpha}(x) \delta_{ \pm 1, e}, \quad \alpha=1, \ldots
$$

we may write the trial state $\Psi_{n}$ as

$$
\Psi_{n}=\prod_{\alpha=1}\left(1-\lambda_{\alpha}^{2}\right)^{1 / 4} \exp \left(-\frac{n}{2}+a^{*}(\phi)-\sum_{e, e^{\prime}= \pm} \sum_{\alpha=1}^{\infty} \frac{\lambda_{\alpha}}{4} e e^{\prime} a_{\alpha e}^{*} a_{\alpha e^{\prime}}^{*}\right)|0\rangle,
$$

where $a_{\alpha, e}^{*}=a\left(\psi_{\alpha e}\right)^{*}$, for $\alpha=1, \ldots$

As discussed in the previous section choosing $n$ and any $\gamma$ with real eigenfunctions uniquely specifies a state $\Psi_{n}$ of the form above (possible degenerate eigenvalues will not 
cause ambiguities). Instead of specifying the individual eigenfunctions $\psi_{\alpha}$ and parameters $\lambda_{\alpha}, \alpha=1, \ldots$ we will simply choose the operator $\gamma$.

The state $\Psi_{n}$ should be compared to Dyson's trial state in [6]. The main difference is that whereas we use a coherent state construction for the condensate, Dyson used squeezed states for this as well. Put differently, Dyson's trial state corresponds to an exponential of a purely quadratic expression in creation operators without any linear terms. As we explained in the previous section the consequence of using the linear term in the exponent is that the variation in the number of particles occupying the state $\phi_{0}$ is much smaller than for a quadratic term.

From (23) we find for the expected number of particles in the state $\Psi_{n}$

$$
\langle\mathcal{N}\rangle=\left\langle\sum_{\alpha=1}^{\infty} \sum_{e= \pm} a_{\alpha e}^{*} a_{\alpha e}\right\rangle=n+\operatorname{Tr} \gamma
$$

and for the kinetic energy expectation

$$
\begin{aligned}
\left(\Psi_{n}, \bigoplus_{N=0}^{\infty} \sum_{i=1}^{N}-\frac{1}{2} \Delta_{i} \Psi_{n}\right) & =\frac{n}{2} \int\left|\nabla \phi_{0}\right|^{2}+\operatorname{Tr}\left(-\frac{1}{2} \Delta \gamma\right) \\
& =\frac{n^{7 / 5}}{2} \int|\nabla \Phi|^{2}+\operatorname{Tr}\left(-\frac{1}{2} \Delta \gamma\right)
\end{aligned}
$$

From (6) we get that

$$
\left(\Psi_{n}, \bigoplus_{N=0}^{\infty} \sum_{1 \leq i<j \leq N} \frac{e_{i} e_{j}}{\left|x_{i}-x_{j}\right|} \Psi_{n}\right)=\frac{1}{2} \sum_{\alpha, \beta, \mu, \nu=1}^{\infty} \sum_{e e^{\prime}= \pm} e e^{\prime} w_{\alpha \beta \nu \mu}\left\langle a_{\alpha e^{*}}^{*} a_{\beta e^{\prime}}^{*} a_{\mu e^{\prime}} a_{\nu e}\right\rangle
$$

where

$$
w_{\alpha \beta \nu \mu}=\iint \psi_{\alpha}(x) \psi_{\beta}(y)|x-y|^{-1} \psi_{\nu}(x) \psi_{\mu}(y) \mathrm{d} x \mathrm{~d} y .
$$

(Since the Coulomb energy is an unbounded operator one may worry about the convergence of the expansion in (31). This problem is easily circumvented by introducing a convergence factor into $|x|^{-1}$, e.g., $|x|^{-1}(1-\exp (-t|x|))$. The expectation on the left of (31) converges as $t \rightarrow \infty$ by the Monotone Convergence Theorem, since for fixed values of the charges each term is monotone in $t$. We may do all calculations and estimates for finite $t$ and at the end let $t \rightarrow \infty$. We will here ignore this slight complication.)

Using the notation of Section 2 we have

$$
\left(\psi_{\beta e^{\prime}}, \gamma_{1} \psi_{\alpha e}\right)=\frac{e e^{\prime}}{2} \frac{\lambda_{\alpha}^{2}}{1-\lambda_{\alpha}^{2}} \delta_{\alpha \beta}, \quad \xi_{1}\left(\psi_{\beta e^{\prime}}, \psi_{\alpha e}\right)=-\frac{e e^{\prime}}{2} \frac{\lambda_{\alpha}}{1-\lambda_{\alpha}^{2}} \delta_{\alpha \beta}
$$


and thus from (24)

$$
\begin{aligned}
& \left\langle a_{\alpha e}^{*} a_{\beta e^{\prime}}^{*} a_{\mu e^{\prime}} a_{\nu e}\right\rangle=\frac{n^{2}}{4}\left(\phi_{0}, \psi_{\alpha}\right)\left(\phi_{0}, \psi_{\beta}\right)\left(\psi_{\mu}, \phi_{0}\right)\left(\psi_{\nu}, \phi_{0}\right) \\
& \quad-n \frac{e e^{\prime}}{4}\left(\delta_{\alpha \beta} \frac{\lambda_{\alpha}}{1-\lambda_{\alpha}^{2}}\left(\psi_{\mu}, \phi_{0}\right)\left(\psi_{\nu}, \phi_{0}\right)+\delta_{\mu \nu} \frac{\lambda_{\mu}}{1-\lambda_{\mu}^{2}}\left(\phi_{0}, \psi_{\alpha}\right)\left(\phi_{0}, \psi_{\beta}\right)\right) \\
& \quad+n \frac{e e^{\prime}}{4}\left(\delta_{\alpha \mu} \frac{\lambda_{\alpha}^{2}}{1-\lambda_{\alpha}^{2}}\left(\phi_{0}, \psi_{\beta}\right)\left(\psi_{\nu}, \phi_{0}\right)+\delta_{\beta \nu} \frac{\lambda_{\beta}^{2}}{1-\lambda_{\beta}^{2}}\left(\phi_{0}, \psi_{\alpha}\right)\left(\psi_{\mu}, \phi_{0}\right)\right) \\
& \quad+\frac{n}{4} \delta_{\beta \mu} \frac{\lambda_{\beta}^{2}}{1-\lambda_{\beta}^{2}}\left(\phi_{0}, \psi_{\alpha}\right)\left(\psi_{\nu}, \phi_{0}\right)+\frac{n}{4} \delta_{\alpha \nu} \frac{\lambda_{\alpha}^{2}}{1-\lambda_{\alpha}^{2}}\left(\phi_{0}, \psi_{\beta}\right)\left(\psi_{\mu}, \phi_{0}\right) \\
& \quad+\frac{\delta_{\alpha \nu} \delta_{\beta \mu}}{4} \frac{\lambda_{\alpha}^{2}}{1-\lambda_{\alpha}^{2}} \frac{\lambda_{\beta}^{2}}{1-\lambda_{\beta}^{2}}+\frac{\delta_{\alpha \mu} \delta_{\beta \nu}}{4} \frac{\lambda_{\alpha}^{2}}{1-\lambda_{\alpha}^{2}} \frac{\lambda_{\beta}^{2}}{1-\lambda_{\beta}^{2}}+\frac{\delta_{\alpha \beta} \delta_{\mu \nu}}{4} \frac{\lambda_{\alpha}}{1-\lambda_{\alpha}^{2}} \frac{\lambda_{\mu}}{1-\lambda_{\mu}^{2}}
\end{aligned}
$$

We therefore arrive at

$$
\left(\Psi_{n}, \bigoplus_{N=0}^{\infty} \sum_{1 \leq i<j \leq N} \frac{e_{i} e_{j}}{\left|x_{i}-x_{j}\right|} \Psi_{n}\right)=\sum_{\alpha=1}^{\infty} w_{\alpha \alpha \mu \nu}\left(\psi_{\nu}, \phi_{0}\right)\left(\psi_{\mu}, \phi_{0}\right) n\left(\frac{\lambda_{\alpha}^{2}}{1-\lambda_{\alpha}^{2}}-\frac{\lambda_{\alpha}}{1-\lambda_{\alpha}^{2}}\right)
$$

where we have used that $\phi_{0}$ and $\psi_{\alpha}, \alpha=1, \ldots$ are real. From the expression for $w_{\alpha \alpha \mu \nu}$ we see that we may write this as

$$
\left(\Psi_{n}, \bigoplus_{N=0}^{\infty} \sum_{1 \leq i<j \leq N} \frac{e_{i} e_{j}}{\left|x_{i}-x_{j}\right|} \Psi_{n}\right)=n \operatorname{Tr}(\mathcal{K}(\gamma-\sqrt{\gamma(\gamma+1)}))
$$

where $\mathcal{K}$ is the operator on $L^{2}\left(\mathbb{R}^{3}\right)$ with integral kernel

$$
\mathcal{K}(x, y)=\phi_{0}(x)|x-y|^{-1} \phi_{0}(y)
$$

Putting together (30) and (35) we arrive at

$$
\left\langle H^{(2)}\right\rangle=\frac{n^{7 / 5}}{2} \int|\nabla \Phi|^{2}+\operatorname{Tr}\left(-\frac{1}{2} \Delta \gamma\right)+n \operatorname{Tr}(\mathcal{K}(\gamma-\sqrt{\gamma(\gamma+1)})) .
$$

Our next goal is to construct the operator $\gamma$. Here we shall use the method of coherent states symbols. Let $\chi(x)=\pi^{-3 / 2} \exp \left(-x^{2}\right)$ such that $\int \chi(x)^{2} \mathrm{~d} x=1$. Let $0<\ell$ be a parameter which we shall specify below as a function of $n$ such that $n^{-2 / 5} \ll \ell \ll n^{-1 / 5}$. Denote $\chi_{\ell}(x)=\ell^{-3 / 2} \chi(x / \ell)$ and let

$$
\theta_{u, p}(x)=\exp (i p x) \chi_{\ell}(x-u)
$$

We then define $\gamma$ to be the operator

$$
\gamma=(2 \pi)^{-3} \iint_{\mathbb{R}^{3} \times \mathbb{R}^{3}} f(u, p)\left|\theta_{u, p}\right\rangle\left\langle\theta_{u, p}\right| \mathrm{d} u \mathrm{~d} p
$$


where

$$
f(u, p)=g\left(\frac{p}{\left(8 \pi n \phi_{0}(u)^{2}\right)^{1 / 4}}\right), \quad \text { where } \quad g(p)=\frac{1}{2}\left(\frac{p^{4}+1}{p^{2}\left(p^{4}+2\right)^{1 / 2}}-1\right) .
$$

We see that $f(u, p) \geq 0$ and hence $\gamma$ is a positive semi-definite operator and since $f(u, p)=$ $f(u,-p)$ all eigenfunctions of $\gamma$ may be chosen real. That this is an appropriate choice for the function $f$ will be seen at the end of our calculation (see (48)). Moreover,

$$
\begin{aligned}
\operatorname{Tr} \gamma & =(2 \pi)^{-3} \iint f(u, p) \mathrm{d} u \mathrm{~d} p=\pi^{-9 / 4}\left(\frac{n}{2}\right)^{3 / 4} \int_{\mathbb{R}^{3}} \phi_{0}(u)^{3 / 2} \mathrm{~d} u \int_{\mathbb{R}^{3}} g(p) \mathrm{d} p \\
& =2^{-3 / 4} \pi^{-9 / 4} n^{3 / 5} \int_{\mathbb{R}^{3}} \Phi(u)^{3 / 2} \mathrm{~d} u \int_{\mathbb{R}^{3}} g(p) \mathrm{d} p .
\end{aligned}
$$

Thus $\gamma$ is a trace class operator. Hence we have all the requirements needed in order for $\gamma$ to define a state $\Psi_{n}$. Moreover, we see from (29) that for large $n$

$$
\langle\mathcal{N}\rangle=n+\mathcal{O}\left(n^{3 / 5}\right)
$$

We turn now to the calculation of the expectation of the kinetic energy.

$$
\begin{aligned}
\operatorname{Tr}(-\Delta \gamma) & =(2 \pi)^{-3} \iiint\left|\nabla \theta_{u, p}\right|^{2} f(u, p) \mathrm{d} u \mathrm{~d} p \\
& =(2 \pi)^{-3} \iint p^{2} f(u, p) \mathrm{d} u \mathrm{~d} p+(2 \pi)^{-3} \int(\nabla \chi)^{2} \ell^{-2} \iint f(u, p) \mathrm{d} u \mathrm{~d} p \\
& \leq(2 \pi)^{-3} \iint p^{2} f(u, p) \mathrm{d} u \mathrm{~d} p+C\left(n^{2 / 5} \ell\right)^{-2} n^{7 / 5} \\
& =2^{3 / 4} \pi^{-7 / 4} n^{7 / 5} \int_{\mathbb{R}^{3}} \Phi(u)^{5 / 2} \mathrm{~d} u \int_{\mathbb{R}^{3}} p^{2} g(p) \mathrm{d} p+C\left(n^{2 / 5} \ell\right)^{-2} n^{7 / 5},
\end{aligned}
$$

where in the second to last inequality we have used the definition (26) of $\phi_{0}$.

The next step in calculating the energy expectation in the state $\Psi_{n}$ is to calculate (or rather estimate) $\operatorname{Tr}(\mathcal{K}(\sqrt{\gamma(\gamma+1)}-\gamma))$. In order to do this we shall use the operator version of the Berezin-Lieb inequality given in (76) in Theorem A.1 in Appendix A We will use it for the operator concave function $\xi(t)=\sqrt{t(t+1)}-t$ (see the discussion at the end of Appendix A and the map $\omega \mapsto|\omega\rangle$ being $(u, p) \mapsto\left|\theta_{u, p}\right\rangle$. We have

$$
(2 \pi)^{-3} \int\left|\theta_{u, p}\right\rangle\left\langle\theta_{u, p}\right| \mathrm{d} u \mathrm{~d} p=I .
$$

Since $\mathcal{K}$ is a positive operator we conclude from Theorem A.1 that

$$
\begin{aligned}
& \operatorname{Tr}(\mathcal{K}(\sqrt{\gamma(\gamma+1)}-\gamma)) \\
& \quad \geq(2 \pi)^{-3} \iint(\sqrt{f(u, p)(f(u, p)+1)}-f(u, p))\left\langle\theta_{u, p}|\mathcal{K}| \theta_{u, p}\right\rangle \mathrm{d} u \mathrm{~d} p
\end{aligned}
$$


Since $|x-y|^{-1}$ is a positive definite kernel we have for $0 \leq \delta^{\prime}$

$$
\begin{aligned}
\left\langle\theta_{u, p}|\mathcal{K}| \theta_{u, p}\right\rangle= & \iint e^{i p x} \chi_{\ell}(x-u) \phi_{0}(x)|x-y|^{-1} e^{-i p y} \chi_{\ell}(y-u) \phi_{0}(y) \mathrm{d} x \mathrm{~d} y \\
\geq & \left(1-C \delta^{\prime}\right) \phi_{0}(u)^{2} \iint e^{i p x} \chi_{\ell}(x-u)|x-y|^{-1} e^{-i p y} \chi_{\ell}(y-u) \mathrm{d} x \mathrm{~d} y \\
& -C \delta^{\prime-1}\left(n^{2 / 5} \ell\right)^{4} n^{-3 / 5} \\
\geq & \phi_{0}(u)^{2} \iint e^{i p x} \chi_{\ell}(x)|x-y|^{-1} e^{-i p y} \chi_{\ell}(y) \mathrm{d} x \mathrm{~d} y-C \delta^{\prime}\left(n^{2 / 5} \ell\right)^{2} n^{-1 / 5} \\
& -C \delta^{\prime-1}\left(n^{2 / 5} \ell\right)^{4} n^{-3 / 5} \\
\geq & \phi_{0}(u)^{2} \int j_{\ell}(q) \frac{4 \pi}{|p-q|^{2}} \mathrm{~d} q-C\left(n^{2 / 5} \ell\right)^{3} n^{-2 / 5},
\end{aligned}
$$

where $j_{\ell}(q)=(2 \pi)^{-3}\left|\widehat{\chi}_{\ell}(q)\right|^{2}=\ell^{3} \pi^{-3} e^{-2 \ell^{2} q^{2}}$ (with the convention $\widehat{f}(p)=\int e^{i p x} f(x) \mathrm{d} x$ for the Fourier transform). In the last inequality we have chosen $\delta^{\prime}=\left(n^{2 / 5} \ell\right) n^{-1 / 5}$ and in the first inequality we have used that $\left|\phi_{0}(x)-\phi_{0}(u)\right| \leq C n^{1 / 2}|x-u|$ and hence

$$
\iint \chi_{\ell}(x-u)\left|\phi_{0}(x)-\phi_{0}(u)\right||x-y|^{-1} \chi_{\ell}(y-u)\left|\phi_{0}(y)-\phi_{0}(u)\right| \mathrm{d} x \mathrm{~d} y \leq C\left(n^{2 / 5} \ell\right)^{4} n^{-3 / 5} .
$$

We have that $\int j_{\ell}(q) \mathrm{d} q=1$. We will use the estimate

$$
\begin{aligned}
& \left.|| p\right|^{-2}-j_{\ell} *|p|^{-2} \mid \\
& \leq|p|^{-2} \int j_{\ell}(q) \frac{|q|}{|p-q|} \mathrm{d} q+|p|^{-1} \int j_{\ell}(q) \frac{|q|}{|p-q|^{2}} \mathrm{~d} q \\
& \leq \sup \left(j_{\ell}(q)|q|^{7 / 2}\right)\left(|p|^{-2} \int|q|^{-5 / 2}|p-q|^{-1} \mathrm{~d} q+|p|^{-1}\left(\int|q|^{-5 / 2}|p-q|^{-2} \mathrm{~d} q\right)\right) \\
& \leq C|p|^{-5 / 2} \sup \left(j_{\ell}(q)|q|^{7 / 2}\right) .
\end{aligned}
$$

For our explicit choice of $j_{\ell}$ we get $\left.|| p\right|^{-2}-\left.j_{\ell} *|p|^{-2}\left|\leq \ell^{-1 / 2}\right| p\right|^{-5 / 2}$. From (44), (45) and estimate (41) we find that

$$
\begin{aligned}
\operatorname{Tr}(\mathcal{K}( & \sqrt{\gamma(\gamma+1)}-\gamma)) \\
\geq & 2(2 \pi)^{-2} \iint(\sqrt{f(u, p)(f(u, p)+1)}-f(u, p)) \phi_{0}(u)^{2} j_{\ell} *|p|^{-2} \mathrm{~d} u \mathrm{~d} p \\
& -C\left(n^{2 / 5} \ell\right)^{3} n^{1 / 5} \\
\geq & 2^{-1 / 4} \pi^{-7 / 4} n^{2 / 5} \iint(\sqrt{g(p)(g(p)+1)}-g(p)) \Phi(u)^{5 / 2}|p|^{-2} \mathrm{~d} u \mathrm{~d} p \\
& -C\left(n^{2 / 5} \ell\right)^{-1 / 2} n^{2 / 5}-C\left(n^{2 / 5} \ell\right)^{3} n^{1 / 5},
\end{aligned}
$$

where we have also used that $\iint(\sqrt{f(u, p)(f(u, p)+1)}-f(u, p)) \mathrm{d} u \mathrm{~d} p \leq C n^{3 / 5}$ (as in (41)). 
If we now insert the above estimate and (43) into (37) we arrive at

$$
\begin{aligned}
\left\langle H^{(2)}\right\rangle \leq n^{7 / 5}( & \frac{1}{2} \int_{\mathbb{R}^{3}}|\nabla \Phi(u)|^{2} \mathrm{~d} u \\
& \left.+2^{-1 / 4} \pi^{-7 / 4} \int_{\mathbb{R}^{3}} \Phi(u)^{5 / 2} \mathrm{~d} u \int_{\mathbb{R}^{3}} p^{2} g(p)-|p|^{-2}(\sqrt{g(p)(g(p)+1)}-g(p)) \mathrm{d} p\right) \\
& +C n^{7 / 5}\left(\left(n^{2 / 5} \ell\right)^{3} n^{-1 / 5}+\left(n^{2 / 5} \ell\right)^{-1 / 2}\right) .
\end{aligned}
$$

The function $g$ in (40) was chosen precisely so as to optimize the above expression. If we insert the expression for $g$ it is easily seen that the term in the large parenthesis above is

$$
\frac{1}{2} \int_{\mathbb{R}^{3}}|\nabla \Phi(u)|^{2} \mathrm{~d} u-I_{0} \int_{\mathbb{R}^{3}} \Phi(u)^{5 / 2} \mathrm{~d} u .
$$

If we choose $\Phi$ to be an exact minimizer then this expression is $-A$ (recall that $A$ and $I_{0}$ were defined in Theorem 1.2). From the estimate in (48) we see that if we choose $\ell$ as a function of $n$ such that $\ell n^{2 / 5}=n^{2 / 35}$ then

$$
\left\langle H^{(2)}\right\rangle \leq-A n^{7 / 5}\left(1-C n^{-1 / 35}\right)
$$

Because of the estimate (42) this means that we have found a state satisfying (25).

We could instead have chosen $\Phi$ to be a smooth compactly supported approximate minimizer to the variational problem (4). We would then for any $\varepsilon>0$ have proved that $\lim _{n \rightarrow \infty} n^{-7 / 5}\left\langle H^{(2)}\right\rangle \leq-A+\varepsilon$, which of course implies (25).

\subsection{An upper bound for fixed particle number}

In this section we shall prove the upper bound in Theorem 1.2 on the energy $E^{(2)}(N)$ corresponding to a fixed particle number $N$.

Let $\Psi_{\varepsilon, n}$ for $n, \varepsilon>0$ denote the state constructed in the previous section, but with the function $g$ in (40) replaced by the function $g_{\varepsilon}$, which is equal to $g$ for $|p|>\varepsilon$ and is zero otherwise. We will again denote the expectation of any operator $\mathcal{A}$ in the state $\Psi_{\varepsilon, n}$, by $\langle\mathcal{A}\rangle$. It then follows from the construction in the previous section that

$$
\lim _{n \rightarrow \infty} n^{-7 / 5}\left\langle H^{(2)}\right\rangle \leq-A_{\varepsilon}
$$

where $A_{\varepsilon} \rightarrow A$ as $\varepsilon \rightarrow 0$.

Let $\Psi_{\varepsilon, n}^{(m)}$ denote the projection of the state $\Psi_{\varepsilon, n}$ onto the subspace corresponding to particle number $m=0,1, \ldots$. We then have

$$
\left\langle\mathcal{N}^{2}\right\rangle=\sum_{m=0}^{\infty} m^{2}\left\|\Psi_{\varepsilon, n}^{(m)}\right\|^{2}=\left\langle\left(\sum_{e= \pm} \sum_{\alpha=1}^{\infty} a_{\alpha e}^{*} a_{\alpha, e}\right)^{2}\right\rangle .
$$


Hence from (29) and (34)

$$
\left\langle\mathcal{N}^{2}\right\rangle-\langle\mathcal{N}\rangle^{2}=\sum_{\alpha=1}^{\infty} \sum_{e, e^{\prime}= \pm}\left\langle a_{\alpha e}^{*} a_{\alpha, e} a_{\alpha e^{\prime}}^{*} a_{\alpha, e^{\prime}}\right\rangle-\left\langle a_{\alpha e}^{*} a_{\alpha, e}\right\rangle\left\langle a_{\alpha e^{\prime}}^{*} a_{\alpha, e^{\prime}}\right\rangle=n+2 \operatorname{Tr} \gamma_{\varepsilon}\left(\gamma_{\varepsilon}+1\right)
$$

where $\gamma_{\varepsilon}$ is given as in (39), but with $f$ replaced by $f_{\varepsilon}$, which is expressed in terms of $g_{\varepsilon}$ instead of $g$. Thus using (75) in Theorem A.1 (or (76) for that matter) in the convex case, we see that

$$
\left\langle\mathcal{N}^{2}\right\rangle-\langle\mathcal{N}\rangle^{2} \leq n+2(2 \pi)^{-3} \iint f_{\varepsilon}(u, p)\left(f_{\varepsilon}(u, p)+1\right) \mathrm{d} u \mathrm{~d} p \leq n+C_{\varepsilon} n^{3 / 5} .
$$

Here $C_{\varepsilon}>0$ is a constant depending on $\varepsilon$ and such that $C_{\varepsilon} \rightarrow \infty$ as $\varepsilon \rightarrow 0$. It is at this point that it is necessary to replace $g$ with $g_{\varepsilon}$, since otherwise the above integral is not convergent.

For any $M>0$ we have

$$
\begin{aligned}
\sum_{m-\langle\mathcal{N}\rangle>M} m^{7 / 5}\left\|\Psi_{\varepsilon, n}^{(m)}\right\|^{2} & \leq M^{-3 / 5} \sum_{m=0}^{\infty} m^{7 / 5}|m-\langle\mathcal{N}\rangle|^{3 / 5}\left\|\Psi_{\varepsilon, n}^{(m)}\right\|^{2} \\
& \leq M^{-3 / 5}\left\langle\mathcal{N}^{2}\right\rangle^{7 / 10}\left\langle(\mathcal{N}-\langle\mathcal{N}\rangle)^{2}\right\rangle^{3 / 10} \\
& =M^{-3 / 5}\left\langle\mathcal{N}^{2}\right\rangle^{7 / 10}\left(\left\langle\mathcal{N}^{2}\right\rangle-\langle\mathcal{N}\rangle^{2}\right)^{3 / 10} \leq C_{\varepsilon} M^{-3 / 5} n^{17 / 10}
\end{aligned}
$$

Given a positive integer $N$, we choose $n=N-C_{0} N^{3 / 5}$. Then if $C_{0}>0$ is chosen appropriately we have according to (29) and (42) that the expected particle number satisfies

$$
N-C_{1} N^{3 / 5} \leq\langle\mathcal{N}\rangle \leq N-C_{2} N^{3 / 5}
$$

for some $C_{1}, C_{2}>0$.

Since $M \mapsto E(M)$ is a non-increasing and non-positive function (adding particles will always lower the energy, since one may construct a trial state with the extra particles placed arbitrarily far away from the original particles) we have that

$$
\begin{aligned}
E^{(2)}(N) \leq \sum_{m \leq N} E^{(2)}(m)\left\|\Psi_{\varepsilon, n}^{(m)}\right\|^{2} & \leq \sum_{m=0}^{\infty} E^{(2)}(m)\left\|\Psi_{\varepsilon, n}^{(m)}\right\|^{2}-\sum_{m>\langle\mathcal{N}\rangle+C_{2} N^{3 / 5}} E^{(2)}(m)\left\|\Psi_{\varepsilon, n}^{(m)}\right\|^{2} \\
& \leq\left\langle H^{(2)}\right\rangle+\sum_{m>\langle\mathcal{N}\rangle+C_{2} N^{3 / 5}} C m^{7 / 5}\left\|\Psi_{\varepsilon, n}^{(m)}\right\|^{2} \\
& \leq\left\langle H^{(2)}\right\rangle+C_{\varepsilon} N^{7 / 5-3 / 50}
\end{aligned}
$$

where we have used the lower bound $E^{(2)}(m) \geq-C m^{7 / 5}$ (see [5] or [1] ) and the estimate (51). Thus we finally get the upper bound in Theorem 1.2

$$
\limsup _{N \rightarrow \infty} N^{-7 / 5} E^{(2)}(N) \leq \lim _{\varepsilon \rightarrow 0} \limsup _{n \rightarrow \infty} n^{-7 / 5}\left(\left\langle H^{(2)}\right\rangle+C_{\varepsilon} N^{7 / 5-3 / 50}\right)=-A,
$$

according to (50). 


\section{The one-component charged Bose gas}

Since the thermodynamic ground state energy $e(\rho)$ of the one-component charged Bose gas may be calculated by minimizing over all particle numbers we may again consider the grand canonical ensemble. Thus we are looking for an upper bound to the ground state energy of the Hamiltonian $H^{(1)}=\bigoplus_{N=0}^{\infty} H_{N}^{(1)}$ acting on the Bosonic Fock space $\mathcal{F}\left(L^{2}(\Lambda)\right)$.

To construct a grand canonical trial function we begin by choosing a real normalized function $\phi_{0} \in L^{2}(\Lambda)$. Let $\eta \in C_{0}^{1}(0, L)$ be a non-negative function compactly supported in $(0, L)$ and such that $\int_{0}^{\infty} \eta(t)^{2} \mathrm{~d} t=1$. Moreover, assume that $\eta(t)$ is a constant for $t \in[r, L-r]$ for some $0<r<L / 4$ to be chosen below. We will write this constant as $(\rho / n)^{1 / 6}$, for some $n>0$. In fact, we shall choose $r$ independently of $L$ (for large $L$ ). We also assume that $\eta(t) \leq(\rho / n)^{1 / 6}$. We then define

$$
\phi_{0}(x, y, z)=\eta(x) \eta(y) \eta(z) .
$$

Thus $\phi_{0}$ is equal to a constant $\sqrt{\rho / n}$ on the cube $[r, L-r]^{3}$ and $0 \leq \phi_{0}(x) \leq \sqrt{\rho / n}$ for all $x \in \Lambda$. Since $\eta$ is normalized so is $\phi_{0}$ and $\rho(L-2 r)^{3} \leq n \leq \rho L^{3}$. Thus the constant $n$ is almost the number of particles required to have a neutral system. We have

$$
|\eta(t)| \leq C L^{-1 / 2} \quad \text { and } \quad\left|\phi_{0}(x)\right| \leq C L^{-3 / 2}
$$

and we may assume that the derivatives satisfy

$$
\left|\eta^{\prime}(t)\right| \leq C r^{-1} L^{-1 / 2} \text { and hence }\left|\nabla \phi_{0}(x)\right| \leq C r^{-1} L^{-3 / 2}
$$

In particular, we have

$$
\int_{\Lambda}\left|\nabla \phi_{0}(x)\right|^{2} \mathrm{~d} x \leq C(r L)^{-1}
$$

Observe that we also have that

$$
\iint\left(n \phi_{0}(x)^{2}-\rho\right)|x-y|^{-1}\left(n \phi_{0}(y)^{2}-\rho\right) \mathrm{d} x \mathrm{~d} y \leq C \rho^{2} L^{3} r^{2} .
$$

We choose our grand canonical trial function $\Psi_{n}$ as in (22). The condensate vector is

$$
\phi=z_{0} \phi_{0}
$$

where the parameter $z_{0}>0$ will be chosen below. The operator $\gamma_{1}=\gamma$ (we omit the subscript 1 because we shall use a subscript $\varepsilon$ below with a different meaning) will be chosen to be a positive semi-definite trace class operator with real eigenfunctions. The eigenfunctions (corresponding to non-zero eigenvalues) should satisfy Dirichlet boundary conditions on the 
boundary of $\Lambda$. Let $\psi_{\alpha}, \alpha=1, \ldots$ be an orthonormal basis of real eigenfunctions for $\gamma$. We use the notation $a_{\alpha}^{*}=a^{*}\left(\psi_{\alpha}\right)$.

As usual we denote the expectation of an operator $\mathcal{A}$ in the state $\Psi_{n}$ by $\langle\mathcal{A}\rangle$. As in (30) we see from (15) and (23)

$$
\left(\Psi_{n}, \bigoplus_{N=0}^{\infty} \sum_{i=1}^{N}-\frac{1}{2} \Delta_{i} \Psi_{n}\right)=\frac{z_{0}^{2}}{2} \int\left|\nabla \phi_{0}\right|^{2}+\operatorname{Tr}\left(-\frac{1}{2} \Delta \gamma\right) \leq C z_{0}^{2}(r L)^{-1}+\operatorname{Tr}\left(-\frac{1}{2} \Delta \gamma\right),
$$

where in the last inequality we have used (55). We likewise get

$$
\begin{aligned}
\left(\Psi_{n}, \bigoplus_{N=0}^{\infty} \sum_{i=1}^{N} V\left(x_{i}\right) \Psi_{n}\right) & =\int V(x) \phi(x)^{2} \mathrm{~d} x+\int V(x) \rho_{\gamma}(x) \mathrm{d} x \\
& =\rho \iint_{\Lambda \times \Lambda} \frac{z_{0}^{2} \phi_{0}(y)^{2}+\rho_{\gamma}(y)}{|x-y|} \mathrm{d} x \mathrm{~d} y,
\end{aligned}
$$

where $\rho_{\gamma}(x)=\gamma(x, x)$ is the density of the operator $\gamma$.

From (6) we have (as in 31) with $w_{\alpha \beta \nu \mu}$ given exactly as in (32)

$$
\left(\Psi_{n}, \bigoplus_{N=0}^{\infty} \sum_{1 \leq i<j \leq N}\left|x_{i}-x_{j}\right|^{-1} \Psi_{n}\right)=\frac{1}{2} \sum_{\alpha, \beta, \mu, \nu=1}^{\infty} w_{\alpha \beta \nu \mu}\left\langle a_{\alpha}^{*} a_{\beta}^{*} a_{\mu} a_{\nu}\right\rangle .
$$

We then obtain from (24) that

$$
\begin{aligned}
& \left(\Psi_{n}, \bigoplus_{N=0}^{\infty} \sum_{1 \leq i<j \leq N}\left|x_{i}-x_{j}\right|^{-1} \Psi_{n}\right)=\frac{z_{0}^{4}}{2} \iint_{\Lambda \times \Lambda} \phi_{0}(x)^{2}|x-y|^{-1} \phi_{0}(y)^{2} \mathrm{~d} x \mathrm{~d} y \\
& \quad+z_{0}^{2} \operatorname{Tr}(\mathcal{K}(\gamma-\sqrt{\gamma(\gamma+1)}))+z_{0}^{2} \iint_{\Lambda \times \Lambda} \phi_{0}(x)^{2}|x-y|^{-1} \rho_{\gamma}(x) \mathrm{d} x \mathrm{~d} y \\
& \quad+\frac{1}{2} \iint_{\Lambda \times \Lambda} \frac{|\gamma(x, y)|^{2}}{|x-y|} \mathrm{d} x \mathrm{~d} y+\frac{1}{2} \iint_{\Lambda \times \Lambda} \frac{|\sqrt{\gamma(\gamma+1)}(x, y)|^{2}}{|x-y|} \mathrm{d} x \mathrm{~d} y \\
& \quad+\frac{1}{2} \iint_{\Lambda \times \Lambda} \rho_{\gamma}(x)|x-y|^{-1} \rho_{\gamma}(y) \mathrm{d} x \mathrm{~d} y,
\end{aligned}
$$

where the operator $\mathcal{K}$ is given as in (36). Putting together (58), (59), and (60) we arrive at

$$
\begin{aligned}
\left\langle H^{(1)}\right\rangle \leq & C z_{0}^{2}(r L)^{-1}+\frac{1}{2} \iint \frac{|\gamma(x, y)|^{2}}{|x-y|} d x d y+\frac{1}{2} \iint \frac{|\sqrt{\gamma(\gamma+1)}(x, y)|^{2}}{|x-y|} \mathrm{d} x \mathrm{~d} y \\
& +\frac{1}{2} \iint_{\Lambda \times \Lambda}\left(\rho-\rho_{\gamma}(x)-z_{0}^{2} \phi_{0}(x)^{2}\right)|x-y|^{-1}\left(\rho-\rho_{\gamma}(y)-z_{0}^{2} \phi_{0}(y)^{2}\right) \mathrm{d} x \mathrm{~d} y \\
& +\operatorname{Tr}\left(-\frac{1}{2} \Delta \gamma\right)+z_{0}^{2} \operatorname{Tr}(\mathcal{K}(\gamma-\sqrt{\gamma(\gamma+1)})) .
\end{aligned}
$$

We now choose

$$
\gamma=\gamma_{\varepsilon}=(2 \pi)^{-3} \int_{\mathbb{R}^{3}} g_{\varepsilon}\left(\frac{p}{(8 \pi \rho)^{1 / 4}}\right)\left|\theta_{p}\right\rangle\left\langle\theta_{p}\right| \mathrm{d} p
$$


where the function $g_{\varepsilon}(p)=0$ for $|p| \leq \varepsilon$ and $g_{\varepsilon}(p)=g(p)$ for $|p|>\varepsilon$ where $g$ is defined in (401), and

$$
\theta_{p}(x)=\sqrt{n \rho^{-1}} \exp (i p x) \phi_{0}(x)
$$

Recall that $n \rho^{-1} \phi_{0}(x)^{2} \leq 1$ and is equal to 1 on most of $\Lambda$.

We see that the map $p \mapsto\left|\theta_{p}\right\rangle$ satisfies the requirements of the map $\omega \mapsto|\omega\rangle$ in Theorem A.1 with measure $d \mu(\omega)=(2 \pi)^{-3} \mathrm{~d} p$.

That $\gamma_{\varepsilon}$ satisfies the necessary requirements follows as before. It is clear that the eigenfunctions of $\gamma_{\varepsilon}$ with non-zero eigenvalues have compact support in $(0, L)^{3}$.

We calculate the density of $\gamma_{\varepsilon}$

$$
\begin{aligned}
\rho_{\gamma_{\varepsilon}}(x) & =(2 \pi)^{-3} \int_{\mathbb{R}^{3}} g_{\varepsilon}\left(\frac{p}{(8 \pi \rho)^{1 / 4}}\right)\left|\theta_{p}(x)^{2}\right| \mathrm{d} p \\
& =(2 \pi)^{-3} n \rho^{-1} \phi_{0}(x)^{2} \int_{\mathbb{R}^{3}} g_{\varepsilon}\left(\frac{p}{(8 \pi \rho)^{1 / 4}}\right) \mathrm{d} p \\
& =n \rho^{-1 / 4} 2^{-3 / 4} \pi^{-9 / 4} \phi_{0}(x)^{2} \int g_{\varepsilon}(p) \mathrm{d} p .
\end{aligned}
$$

We finally choose $z_{0}>0$

$$
z_{0}^{2}=n\left(1-2^{-3 / 4} \rho^{-1 / 4} \pi^{-9 / 4} \int g_{\varepsilon}(p) \mathrm{d} p\right)
$$

(for $\rho$ large enough). Then

$$
z_{0}^{2} \phi_{0}(x)^{2}+\rho_{\gamma_{\varepsilon}}(x)=n \phi_{0}(x)^{2} .
$$

It follows from (56) and the fact that $\phi_{0}(x)^{2} \leq \rho / n$ that

$$
\iint_{\Lambda \times \Lambda}\left(\rho-\rho_{\gamma_{\varepsilon}}(x)-z_{0}^{2} \phi_{0}(x)^{2}\right)|x-y|^{-1}\left(\rho-\rho_{\gamma_{\varepsilon}}(y)-z_{0}^{2} \phi_{0}(y)^{2}\right) \mathrm{d} x \mathrm{~d} y \leq C \rho^{2} L^{3} r^{2} .
$$

To estimate the second term in (61) we will use Hardy's inequality $\int|\nabla u(x)|^{2} \mathrm{~d} x \geq$ $\frac{1}{4} \int \frac{|u(x)|}{|x|^{2}} \mathrm{~d} x$ as follows

$$
\begin{aligned}
\iint \frac{\left|\gamma_{\varepsilon}(x, y)\right|^{2}}{|x-y|} \mathrm{d} x \mathrm{~d} y & \leq\left(\iint\left|\gamma_{\varepsilon}(x, y)\right|^{2} \mathrm{~d} x \mathrm{~d} y\right)^{1 / 2}\left(\iint \frac{\left|\gamma_{\varepsilon}(x, y)\right|^{2}}{|x-y|^{2}} \mathrm{~d} x \mathrm{~d} y\right)^{1 / 2} \\
& \leq 2\left(\iint\left|\gamma_{\varepsilon}(x, y)\right|^{2} \mathrm{~d} x \mathrm{~d} y\right)^{1 / 2}\left(\iint\left|\nabla_{x} \gamma_{\varepsilon}(x, y)\right|^{2} \mathrm{~d} x \mathrm{~d} y\right)^{1 / 2} \\
& =2\left(\operatorname{Tr} \gamma_{\varepsilon}^{2}\right)^{1 / 2}\left(\operatorname{Tr}\left(-\Delta \gamma_{\varepsilon}^{2}\right)\right)^{1 / 2}
\end{aligned}
$$

Since $x \mapsto x^{2}$ is operator convex we may estimate these terms using the Berezin-Lieb inequality (76) in the convex case, but we may alternatively simply use the norm bound $\left\|\gamma_{\varepsilon}\right\| \leq C \varepsilon^{-2}$. Hence

$$
\iint \frac{\left|\gamma_{\varepsilon}(x, y)\right|^{2}}{|x-y|} \mathrm{d} x \mathrm{~d} y \leq C \varepsilon^{-2}\left(\int \rho_{\gamma_{\varepsilon}}(x)\right)^{1 / 2}\left(\operatorname{Tr}\left(-\Delta \gamma_{\varepsilon}\right)\right)^{1 / 2} \leq C \varepsilon^{-2} \rho L^{3},
$$


where we have used (64), $n \leq \rho L^{3}$ and the, fact which we shall prove below in (68), that $\operatorname{Tr}\left(-\Delta \gamma_{\varepsilon}\right) \leq C \rho^{5 / 4} L^{3}$ (recall that we will choose $r$ independently of $L$ ). The third term in (61) which compared to the second term has $\gamma_{\varepsilon}$ replaced by $\sqrt{\gamma_{\varepsilon}\left(\gamma_{\varepsilon}+1\right)}$ is estimated in exactly the same way and with the same bound as the second term.

We are now left with calculating the last two terms in (61). For the kinetic energy of $\gamma_{\varepsilon}$ we have as in (43)

$$
\begin{aligned}
\operatorname{Tr}\left(-\Delta \gamma_{\varepsilon}\right) & \leq(2 \pi)^{-3} \frac{n}{\rho} \int_{\mathbb{R}^{3}} g_{\varepsilon}\left(\frac{p}{(8 \pi \rho)^{1 / 4}}\right)\left(p^{2}+\int\left|\nabla \phi_{0}(x)\right|^{2} \mathrm{~d} x\right) \mathrm{d} p \\
& \leq 2^{3 / 4} \pi^{-7 / 4} \rho^{5 / 4} L^{3} \int_{\mathbb{R}^{3}} p^{2} g_{\varepsilon}(p) \mathrm{d} p+C \rho^{3 / 4} L^{3}(r L)^{-1 / 2},
\end{aligned}
$$

where we have used (55) and $n \leq \rho L^{3}$.

For the last term in (61) we again, as in (44), appeal to the operator version (76) of the Berezin-Lieb inequalities. We arrive at

$$
\begin{aligned}
& \operatorname{Tr}\left(\mathcal{K}\left(\gamma_{\varepsilon}-\sqrt{\gamma_{\varepsilon}\left(\gamma_{\varepsilon}+1\right)}\right)\right) \\
& \leq(2 \pi)^{-3} \int_{\mathbb{R}^{3}}\left(f_{\varepsilon}(p)-\sqrt{f_{\varepsilon}(p)\left(f_{\varepsilon}(p)+1\right)}\right)\left\langle\theta_{p} \mid \mathcal{K} \| \theta_{p}\right\rangle \mathrm{d} p
\end{aligned}
$$

where $f_{\varepsilon}(p)=g_{\varepsilon}\left(p(8 \pi \rho)^{-1 / 4}\right)$. We have as in (45)

$$
\left\langle\theta_{p}|\mathcal{K}| \theta_{p}\right\rangle=4 \pi J *|p|^{-2}
$$

where $J(p)=(2 \pi)^{-3} n \rho^{-1}\left|\widehat{\phi_{0}^{2}}(p)\right|^{2}$. The special form (52) implies that

$$
J\left(p_{1}, p_{2}, p_{3}\right)=j\left(p_{1}\right) j\left(p_{2}\right) j\left(p_{3}\right),
$$

where $j(\tau)=(2 \pi)^{-1} n^{1 / 3} \rho^{-1 / 3}\left|\widehat{\eta^{2}}(\tau)\right|^{2}$. Since $\int j(\tau) \mathrm{d} \tau=n^{1 / 3} \rho^{-1 / 3} \int \eta(t)^{4} \mathrm{~d} t, \int \eta^{2}=1$, and $0 \leq \eta(t) \leq n^{-1 / 3} \rho^{1 / 3}$ and equal to this constant on $[r, L-r]$ we have that $1-2 r / L \leq$ $\int j(\tau) \mathrm{d} \tau \leq 1$. This implies in particular that

$$
(1-2 r / L)^{3} \leq \int J(p) \mathrm{d} p \leq 1
$$

By (153) and (54) and the support property of $\eta^{\prime}$ we we have $\left|\widehat{\eta^{2}}(\tau)\right| \leq|\tau|^{-1} \int\left|\left(\eta^{2}\right)^{\prime}(t)\right| \mathrm{d} t \leq$ $C(|\tau| L)^{-1}$. Thus $j(\tau) \leq C L(|\tau| L)^{-2}$. Hence

$$
\int_{|q|>L^{-1 / 2}} J(q) \mathrm{d} q \leq 3 \int_{|\tau|>(3 L)^{-1 / 2}} j(\tau) \mathrm{d} \tau \leq C L^{-1 / 2} .
$$

For $|p|>\varepsilon(8 \pi \rho)^{1 / 4}$ and $|q| \leq L^{-1 / 2}$ we have $|p-q| \leq\left(1+C \rho^{-1 / 4} \varepsilon^{-1} L^{-1 / 2}\right)|p|$ and hence from (71) and (72)

$$
\begin{aligned}
J *|p|^{-2} & \geq\left(1+C \rho^{-1 / 4} \varepsilon^{-1} L^{-1 / 2}\right)^{-2}|p|^{-2} \int_{|q|<L^{-1 / 2}} J(q) \mathrm{d} q \\
& \geq\left(1+C \rho^{-1 / 4} \varepsilon^{-1} L^{-1 / 2}\right)^{-2}\left(\left(1-2 r L^{-1}\right)^{3}-C L^{-1 / 2}\right)|p|^{-2} \\
& \geq\left(1-C\left(\rho^{-1 / 4} \varepsilon^{-1} L^{-1 / 2}+r L^{-1}+L^{-1 / 2}\right)\right)|p|^{-2}
\end{aligned}
$$


Inserting this into (70) and then into (69) we arrive at

$$
\begin{aligned}
\operatorname{Tr} & \left.\mathcal{K}\left(\gamma_{\varepsilon}-\sqrt{\gamma_{\varepsilon}\left(\gamma_{\varepsilon}+1\right)}\right)\right) \\
\leq & 2^{-1 / 4} \rho^{1 / 4} \pi^{-7 / 4} \int\left(g_{\varepsilon}(p)-\sqrt{g_{\varepsilon}(p)\left(g_{\varepsilon}(p)+1\right)}\right)|p|^{-2} \mathrm{~d} p \\
\quad & +C\left(\varepsilon^{-1} L^{-1 / 2}+\rho^{1 / 4} r L^{-1}+\rho^{1 / 4} L^{-1 / 2}\right) .
\end{aligned}
$$

If we now insert the above estimate, (65), (66), (68), (67), and the same estimate for $\gamma_{\varepsilon}$ replaced by $\left.\sqrt{\gamma_{\varepsilon}\left(\gamma_{\varepsilon}+1\right)}\right)$ into (61) we see that

$$
\begin{aligned}
\limsup _{L \rightarrow \infty} L^{-3}\left\langle H^{(1)}\right\rangle \leq & \rho^{5 / 4} 2^{-1 / 4} \pi^{-7 / 4} \int|p|^{2} g_{\varepsilon}(p)+g_{\varepsilon}(p)|p|^{-2}-\sqrt{g_{\varepsilon}(p)\left(g_{\varepsilon}(p)+1\right)}|p|^{-2} \mathrm{~d} p \\
& +C \rho\left(1+\rho r^{2}+\varepsilon^{-2}\right) .
\end{aligned}
$$

Here we may actually let $r \rightarrow 0$ (which really means that we could have chosen $r$ as a negative power of $L$ ). If we recall the behavior of $g(p)$ for small $|p|$ from (40) we find that the error in replacing $g_{\varepsilon}$ by $g$ is of order $\rho^{5 / 4} \varepsilon$. Thus by choosing $\varepsilon=\rho^{-1 / 12}$ we obtain the final result

$$
e(\rho) \leq \limsup _{L \rightarrow \infty} L^{-3}\left\langle H^{(1)}\right\rangle \leq-I_{0} \rho^{5 / 4}\left(1-C \rho^{-1 / 12}\right) .
$$

\section{A The Berezin-Lieb inequality}

In this appendix we shall prove variants of the Berezin-Lieb inequalities [2, 8].

THEOREM A.1 (Berezin-Lieb inequalities). Let $\mathcal{H}$ be a Hilbert space and $\Omega$ a measure space with a (positive) measure $\mu$ such that there exists a map

$$
\Omega \ni \omega \mapsto|\omega\rangle \in \mathcal{H}
$$

satisfying $\int|\omega\rangle\langle\omega| d \mu(\omega) \leq I$ as operators. Assume $\xi: \mathbb{R}_{+} \cup\{0\} \rightarrow \mathbb{R}$ is a concave function with $\xi(0) \geq 0$. Then for any non-negative function $f$ on $\Omega$ satisfying $\int f(\omega)\langle\omega \mid \omega\rangle d \mu(\omega)<\infty$ we have the Berezin-Lieb inequality

$$
\operatorname{Tr}_{\mathcal{H}}\left(\xi\left(\int f(\omega)|\omega\rangle\langle\omega| d \mu(\omega)\right)\right) \geq \int \xi(f(\omega))\langle\omega \mid \omega\rangle d \mu(\omega)
$$

If moreover $\xi$ is operator concave (still satisfying $\xi(0) \geq 0$ ) the inequality holds as an operator inequality

$$
\xi\left(\int f(\omega)|\omega\rangle\langle\omega| d \mu(\omega)\right) \geq \int \xi(f(\omega))|\omega\rangle\langle\omega| d \mu(\omega) .
$$


Proof. We first note that $\int f(\omega)|\omega\rangle\langle\omega| d \mu(\omega)$ is a positive semi-definite trace class operator. Let $u_{1}, u_{2}, \ldots$ be an orthonormal basis of eigenvectors for this operator. Then

$$
\begin{aligned}
\operatorname{Tr}_{\mathcal{H}}\left(\xi\left(\int f(\omega)|\omega\rangle\langle\omega| d \mu(\omega)\right)\right) & =\sum_{i=1}^{\infty} \xi\left(\int f(\omega)\left|\left\langle\omega \mid u_{i}\right\rangle\right|^{2} d \mu(\omega)\right) \\
\geq & \sum_{i=1}^{\infty} \int\left|\left\langle\omega \mid u_{i}\right\rangle\right|^{2} d \mu(\omega) \\
& \times \xi\left(\left(\int\left|\left\langle\omega \mid u_{i}\right\rangle\right|^{2} d \mu(\omega)\right)^{-1} \int f(\omega)\left|\left\langle\omega \mid u_{i}\right\rangle\right|^{2} d \mu(\omega)\right),
\end{aligned}
$$

where we have used that $\int\left|\left\langle\omega \mid u_{i}\right\rangle\right|^{2} d \mu(\omega) \leq 1$ and that since $\xi$ is concave with $\xi(0) \geq 0$ we have $\xi(a t) \geq a \xi(t)$ for all $t \geq 0$ and $0<a<1$. If we now use Jensen's inequality we arrive at

$$
\begin{aligned}
\operatorname{Tr}_{\mathcal{H}}\left(\xi\left(\int f(\omega)|\omega\rangle\langle\omega| d \mu(\omega)\right)\right) & \geq \sum_{i=1}^{\infty} \int \xi(f(\omega))\left|\left\langle\omega \mid u_{i}\right\rangle\right|^{2} d \mu(\omega) \\
& =\int \xi(f(\omega))\langle\omega \mid \omega\rangle d \mu(\omega) .
\end{aligned}
$$

We turn to the case when $\xi$ is operator concave. Define the operator $U: \mathcal{H} \rightarrow L^{2}(\Omega, d \mu)$ by $(U \phi)(\omega)=\langle\omega \mid \phi\rangle$. Then

$$
U^{*} h=\int h(\omega)|\omega\rangle d \mu(\omega)
$$

Thus if $B$ is the multiplication operator on $L^{2}(\Omega, d \mu)$ given by $B h(\omega)=f(\omega) h(\omega)$ we have

$$
U^{*} B U=\int f(\omega)|\omega\rangle\langle\omega| d \mu(\omega)
$$

In particular, we have the operator inequalities $0 \leq U^{*} U \leq I$. Using that $\left(1-U U^{*}\right)^{1 / 2} U=$ $U\left(1-U^{*} U\right)^{1 / 2}$ it is straightforward to check that the following operators on $\mathcal{H} \oplus L^{2}(\Omega, d \mu)$ (written in matrix notation) are unitary

$$
\mathcal{U}=\left(\begin{array}{cc}
\left(I-U^{*} U\right)^{1 / 2} & -U^{*} \\
U & \left(I-U U^{*}\right)^{1 / 2}
\end{array}\right), \quad \mathcal{V}=\left(\begin{array}{cc}
\left(I-U^{*} U\right)^{1 / 2} & U^{*} \\
U & -\left(I-U U^{*}\right)^{1 / 2}
\end{array}\right)
$$

Moreover we have that

$$
\frac{1}{2} \mathcal{U}^{*}\left(\begin{array}{cc}
0 & 0 \\
0 & B
\end{array}\right) \mathcal{U}+\frac{1}{2} \mathcal{V}^{*}\left(\begin{array}{cc}
0 & 0 \\
0 & B
\end{array}\right) \mathcal{V}=\left(\begin{array}{cc}
U^{*} B U & 0 \\
0 & \left(1-U U^{*}\right)^{1 / 2} B\left(1-U U^{*}\right)^{1 / 2}
\end{array}\right)
$$


Since $\xi$ is operator concave and $\mathcal{U}$ and $\mathcal{V}$ are unitary we find that

$$
\begin{gathered}
\left(\begin{array}{cc}
\xi\left(U^{*} B U\right) & 0 \\
0 & \xi\left(\left(1-U U^{*}\right)^{1 / 2} B\left(1-U U^{*}\right)^{1 / 2}\right)
\end{array}\right) \\
\geq \frac{1}{2} \mathcal{U}^{*}\left(\begin{array}{cc}
0 & 0 \\
0 & \xi(B)
\end{array}\right) \mathcal{U}+\frac{1}{2} \mathcal{V}^{*}\left(\begin{array}{cc}
0 & 0 \\
0 & \xi(B)
\end{array}\right) \mathcal{V} \\
=\left(\begin{array}{cc}
U^{*} \xi(B) U & 0 \\
0 & \left(1-U U^{*}\right)^{1 / 2} \xi(B)\left(1-U U^{*}\right)^{1 / 2}
\end{array}\right) .
\end{gathered}
$$

In particular, this gives $\xi\left(U^{*} B U\right) \geq U^{*} \xi(B) U$, which is precisely the operator Berezin-Lieb inequality (76).

In order to determine whether a given function is operator concave we may use Nevanlinna's Theorem (see [3] Theorems V.4.11 and V.4.14 and equation (V.49)). According to this a real function $\xi$ defined on the positive real axis with an analytic extension to $\mathbb{C} \backslash\{x \in \mathbb{R} \mid x \leq 0\}$, which maps the upper half plane into itself has a representation of the form

$$
\xi(t)=\alpha+\beta t+\int_{0}^{\infty}\left(\frac{\lambda}{\lambda^{2}+1}-\frac{1}{\lambda+t}\right) d \nu(\lambda)
$$

where $\beta \geq 0$ and where $\nu$ is a positive measure satisfying $\int_{0}^{\infty} \frac{1}{1+\lambda^{2}} d \nu(\lambda)<\infty$. Since $t \mapsto-(t+\lambda)^{-1}$ is operator concave the same is true for functions with the above integral representation.

As a special case we see that the function $\xi(t)=\sqrt{t(t+1)}$, which is analytic away from the segment $[-1,0]$ is operator concave.

\section{References}

[1] V. Bach, E.H. Lieb, J.P. Solovej Generalized Hartree-Fock theory and the Hubbard model, J. Stat. Phys., 76, 3-90 (1994).

[2] F.A. Berezin, Izv. Akad. Nauk, ser. mat., 36 (No. 5) (1972); English translation: USSR Izv. 6 (No. 5) (1972). F.A. Berezin, General concept of quantization, Commun. Math. Phys. 40, 153-174 (1975).

[3] R. Bhatia, Matrix Analysis, Graduate Texts in Mathematics, Vol. 169. Springer-Verlag, New York, (1997).

[4] N.N. Bogolubov, J. Phys. (U.S.S.R.) 11, 23 (1947); N.N. Bogolubov, D.N. Zubarev, Sov. Phys.-JETP 1, 83 (1955). 
[5] J. Conlon, E.H. Lieb, H.-T. Yau, The $N^{7 / 5}$ Law for Charged Bosons, Commun. Math. Phys. 116, 417-448 (1988).

[6] F.J. Dyson, Ground State Energy of a Finite System of Charged Particles, J. Math. Phys. 8, 1538-1545 (1967).

[7] L.L. Foldy, Charged Boson Gas, Phys. Rev. 124, 649-651 (1961); Errata ibid 125, 2208 (1962).

[8] E.H. Lieb, The classical limit of quantum spin systems, Commun. Math. Phys. 31, 327-340 (1973).

[9] E.H. Lieb, H. Narnhofer, The Thermodynamic Limit for Jellium, J. Stat. Phys. 12, 291-310 (1975). Errata J. Stat. Phys. 14, 465 (1976).

[10] E.H. Lieb, J.P. Solovej, Ground State Energy of the One-Component Charged Bose Gas, Commun. Math. Phys. 217, 127-163 (2001). Errata 225, 219-221 (2002).

[11] E.H. Lieb, J.P. Solovej, Ground State Energy of the Two-Component Charged Bose Gas, Commun. Math. Phys. 252, 485-534, (2004). 